\title{
OPTIMAL REES MATRIX CONSTRUCTIONS FOR ANALYSIS OF DATA
}

\author{
A. V. KELAREV ${ }^{凶}$, J. L. YEARWOOD and LIFANG ZI \\ (Received 6 January 2011; accepted 20 September 2011; first published online 8 December 2011) \\ Communicated by M. G. Jackson
}

\begin{abstract}
We introduce a new construction involving Rees matrix semigroups and max-plus algebras that is very convenient for generating sets of centroids. We describe completely all optimal sets of centroids for all Rees matrix semigroups without any restrictions on the sandwich matrices.
\end{abstract}

2010 Mathematics subject classification: primary 20M25; secondary 62H30.

Keywords and phrases: Rees matrix semigroups, sets of centroids, classification and clustering.

\section{Introduction}

Rees matrix semigroups and max-plus algebras are well known and widely used; see $[1,9]$. We describe a new construction that combines Rees matrix semigroups and max-plus algebras. This construction is very effective for generating sets of centroids, which are used in data analysis for the design of centroid-based classifiers or clusterers, as well as for the design of multiple classifiers and clusterers combining several individual initial classifiers and clusterers. We describe all optimal sets of centroids of arbitrary Rees matrix semigroups without any restrictions on the sandwich matrices.

The paper is organised as follows. We include the necessary background information in Section 2. As motivation for our research, we give an overview of applications of the Rees matrix constructions in classification and clustering for analysis of data in Section 3. The main result of this paper is Theorem 4.1, which completely describes all optimal sets of centroids. Proofs are given in Section 5.

The first-named author was supported by Discovery Grant DP0449469 from the Australian Research Council. The second-named author was supported by a Queen Elizabeth II Fellowship, Discovery Grant DP0211866, and Linkage Grant LP0990908 from the Australian Research Council.

(C) 2011 Australian Mathematical Publishing Association Inc. 1446-7887/2011 \$16.00 


\section{Preliminaries}

We begin with brief preliminaries on Rees matrix semigroups required for our main theorem. Rees matrix semigroups and the associated notions of completely 0-simple semigroups and Rees quotients are well known in semigroup theory and play crucial roles in describing the structure of semigroups and in proofs; see [9]. For examples of recent results, we also refer to [4, 10-12].

Suppose that $G$ is a group, $I$ and $\Lambda$ are nonempty sets, and $e$ is the identity of $G$. We write $G^{0}$ for the set $G \cup\{\theta\}$, that is, the group $G$ with zero $\theta$ adjoined. Let $P$ or $\left[p_{\lambda i}\right]$ be a $(\Lambda \times I)$ matrix with entries $p_{\lambda i} \in G^{0}$, where $\lambda \in \Lambda$ and $i \in I$. The Rees matrix semigroup $M^{0}(G ; I, \Lambda ; P)$ over $G$ with sandwich matrix $P$ consists of all triples $(g ; i, \lambda)$, where $g \in G^{0}, i \in I$, and $\lambda \in \Lambda$; all triples $(\theta ; i, \lambda)$ are identified with $\theta$, and multiplication is defined by the rule

$$
\left(g_{1} ; i_{1}, \lambda_{1}\right)\left(g_{2} ; i_{2}, \lambda_{2}\right)=\left(g_{1} p_{\lambda_{1} i_{2}} g_{2} ; i_{1}, \lambda_{2}\right) .
$$

If $G$ is a group, $M=M^{0}(G ; I, \Lambda ; P)$, and $i \in I, \lambda \in \Lambda$, then we use the following standard notation:

$$
\begin{gathered}
G_{* \lambda}=\{(g ; i, \lambda): g \in G, i \in I\}, \\
G_{i *}=\{(g ; i, \lambda): g \in G, \lambda \in \Lambda\}, \\
G_{i \lambda}=\{(g ; i, \lambda): g \in G\} .
\end{gathered}
$$

Further, let $S$ be a subset of the Rees matrix semigroup $M^{0}(G ; I, \Lambda ; P)$. For $i, \lambda \in I$, set

$$
\begin{aligned}
S_{i \lambda} & =S \cap G_{i \lambda}, \\
S_{* \lambda} & =S \cap G_{* \lambda}, \\
S_{i *} & =S \cap G_{i *} .
\end{aligned}
$$

For subsets $X \subseteq I, Y \subseteq \Lambda$, we put

$$
\begin{aligned}
& S_{X *}=\bigcup_{i \in X} S_{i *}, \\
& S_{* Y}=\bigcup_{\lambda \in Y} S_{* \lambda} .
\end{aligned}
$$

We assume that $S_{\emptyset *}=S_{* \emptyset}=\emptyset$. Note that $\theta$ never belongs to any of these sets. For a subset $X$ of $T$, we put $X^{0}=X \cup\{\theta\}$.

The max-plus algebra is the set $\mathbb{R} \cup\{-\infty\}$ with two binary operations, max and + . It is important in the investigation of discrete event systems (see [1]). The max-plus algebra is also sometimes called the schedule algebra (see [8]).

Our main results remain valid in the more general case of idempotent semifields, and so we record them in this setting.

A semiring is a set $F$ with two binary operations, addition + and multiplication $\cdot$ such that the following conditions are satisfied.

(S1) $(F,+)$ is a commutative semigroup with zero 0 .

(S2) $(F, \cdot)$ is a semigroup. 
(S3) Multiplication distributes over addition.

(S4) The zero annihilates $F$, that is, $0 \cdot F=F \cdot 0=0$.

It is also often assumed that every semiring satisfies an additional property.

(S5) $(F, \cdot)$ has an identity element 1 .

In this paper, we consider more general semirings, which do not have to satisfy (S5), since this adds the convenience of allowing us to consider more general subsets as subsemirings. In analogy with the terminology in ring theory, we call a semiring satisfying (S5) a semiring with identity element. The two terminologies are essentially equivalent, since it is always easy to adjoin an identity element in a standard fashion to every semiring that does not have one. Originally, our investigation of semirings was motivated by the development of methods useful for duality theory; see [3, 6].

A semiring $F$ is said to be idempotent if $x+x=x$ for all $x \in F$. If the set of nonzero elements of a semiring $F$ forms a group with respect to multiplication, then $F$ is called a semifield.

Let $F$ be a semiring and let $S$ be a semigroup. The semigroup semiring is denoted by $F[S]$ and is defined by

$$
F[S]=\left\{\sum_{i=1}^{n} f_{i} s_{i}: f_{i} \in F, s_{i} \in S, n \in \mathbb{N}\right\},
$$

where $\mathbb{N}=\{1,2,3, \ldots\}$, and addition and multiplication are defined by the associative and distributive laws and the rules

$$
\begin{gathered}
\sum_{s \in S} f_{s} s+\sum_{s \in S} f_{s}^{\prime} s=\sum_{s \in S}\left(f_{s}+f_{s}^{\prime}\right) s, \\
\left(\sum_{s \in S} r_{s} s\right)\left(\sum_{t \in S} r_{t}^{\prime} t\right)=\sum_{s, t \in S}\left(r_{s} r_{t}^{\prime}\right) s t .
\end{gathered}
$$

If $S$ has a zero $\theta$, then the quotient semiring of $F[S]$ modulo the ideal $F \theta$ is called a contracted semigroup semiring and is denoted by $F_{0}[S]$. If $S$ has no zero, then $S^{0}$ stands for the semigroup $S \cup\{\theta\}$ with zero $\theta$ adjoined, and $F[S]$ is isomorphic to $F_{0}\left[S^{0}\right]$. If $S$ is a semigroup without zero, then we also let $F_{0}[S]=F_{0}\left[S^{0}\right] \cong F[S]$. The use of contracted semigroup semirings enables us to formulate main results more concisely. We refer to [7, 13-19] for examples of results using these constructions and other areas where they are used.

\section{Motivation}

The design of efficient classifiers and clusterers is very important in data mining; see [20]. Rees matrix semigroups can be used to generate convenient sets of centroids for centroid-based clusterers and to design combined multiple clusterers capable of correcting the errors of individual initial clusterers.

The clustering process begins with feature extraction and representation of data in a standard vector space $F^{n}$, where $n \in \mathbb{N}$ and $F$ may be regarded as a semifield. Every centroid-based clusterer selects special elements $c_{1}, \ldots, c_{k}$ in $F^{n}$, called centroids 
(see, for example, [2]). When $i=1, \ldots, k$, each centroid $c_{i}$ defines its cluster $N\left(c_{i}\right)$, which consists of all vectors $v$ such that $c_{i}$ is the nearest centroid of $v$. Every vector is assigned to the cluster of its nearest centroid.

Multiple classifiers and clusterers are often used in analysis of data to combine individual initial classifiers or clusterers (see, for example, [5, 21]). A well-known method for the design of multiple clusterers consists in designing several simpler initial or individual clusterers and then combining them into one multiple clustering scheme with several clusters. This method is very effective and is recommended for various applications (see [20, Section 7.5]). The main advantage of using combined multiple clusterers is in their ability to correct the errors of individual clusterers.

Denote the number of initial clusterers being combined by $n$. The output of each clusterer is a symbol that indicates the cluster of the current instance. Without loss of generality, we may assume that all these outputs belong to the same semifield $F$, because it is possible to extend the semifield when necessary. If $o_{1}, \ldots, o_{n}$ are the outputs of the initial clusterers, then the sequence $\left(o_{1}, \ldots, o_{n}\right)$ is called a vector of outputs of the initial clusterers. In order to define the multiple clusterers and enable correction of errors of the initial clusterers, a set of centroids $c_{1}, \ldots, c_{k}$ is again selected in $F^{n}$. When $i=1, \ldots, k$, the cluster $N\left(c_{i}\right)$ of the centroid $c_{i}$ is again defined as the set of all observations with the vector outputs of the initial clusterers having $c_{i}$ as its nearest centroid.

The design of multiple clusterers by combining individual binary clusterers is quite common in the literature. We refer to $[16,20]$ for a list of properties required of the sets of centroids. In particular, it is essential to find sets of centroids with large weights and small numbers of generators. The weight $\mathrm{wt}(v)$ of $v$ in $F^{n}$ is the number of nonzero components $v$. The weight of a subset $C$ of $F^{n}$ is the minimum weight of a nonzero element in $C$. For additional references and discussion of experimental research related to these properties, we refer to [16], which treats constructions with certain restrictions on the sandwich matrices.

Suppose that $S$ is a finite semigroup with $n$ nonzero elements. Then the additive semigroup of $F_{0}[S]$ is isomorphic to $F^{n}$, and we can introduce multiplication in $F^{n}$ by identifying it with $F_{0}[S]$. Accordingly, we further consider sets of centroids as subsets generated in $F_{0}[S]$. Every set of elements $g_{1}, \ldots, g_{k} \in F_{0}[S]$ generates the set $C\left(g_{1}, \ldots, g_{k}\right)$ of all sums of multiples of these elements:

$$
\left\{\sum_{j=1}^{m_{1}} \ell_{1, j} g_{1} r_{1, j}+\cdots+\sum_{j=1}^{m_{k}} \ell_{k, j} g_{k} r_{k, j}: \ell_{i, j}, r_{i, j} \in F_{0}[S] \cup\{1\}\right\} .
$$

The set $C\left(g_{1}, \ldots, g_{k}\right)$ is also often called the ideal generated by $g_{1}, \ldots, g_{k}$.

\section{Main results}

Let $S$ be a subsemigroup of a Rees matrix semigroup $M^{0}(G ; I, \Lambda ; P)$ over a group $G$ with sandwich matrix $P$. Consider the sets $L(S)$ and $R(S)$, which may be abbreviated 
to $L$ and $R$ :

$$
\begin{aligned}
& L(S)=\left\{\lambda \in \Lambda: S_{* \lambda}=\emptyset \text { or } \bigcup_{i \in I} p_{\lambda i} S_{i *} \subseteq\{\theta\}\right\}, \\
& R(S)=\left\{i \in I: S_{i *}=\emptyset \text { or } \bigcup_{\lambda \in \Lambda} S_{* \lambda} p_{\lambda i} \subseteq\{\theta\}\right\} .
\end{aligned}
$$

Here $p_{\lambda i} S_{i *}=\left\{\left(p_{\lambda i} g ; i, \mu\right):(g ; i, \mu) \in S_{i *}\right\}$, and so $p_{\lambda i} S_{i *} \subseteq\{\theta\}$ means that $p_{\lambda i}=\theta$ or $S_{i *}=\emptyset$. Likewise, $S_{* \lambda} p_{\lambda i}=\left\{\left(g p_{\lambda i} ; i, \mu\right):(g ; i, \mu) \in S_{i *}\right\}$, and so $S_{* \lambda} p_{\lambda i} \subseteq\{\theta\}$ means that $p_{\lambda i}=\theta$ or $S_{* \lambda}=\emptyset$.

We define the following numbers:

$$
\begin{gathered}
M_{Z}=\left|S_{R^{*}} \cap S_{* L}\right|, \\
M_{L}=\max \left\{\left|S_{i *} \cap S_{* L}\right|: i \notin L\right\}, \\
M_{R}=\max \left\{\left|S_{* \lambda} \cap S_{R *}\right|: \lambda \notin R\right\}, \\
M_{G}=\max \left\{\left|S_{i \lambda}\right|: i \notin L, \lambda \notin R\right\} .
\end{gathered}
$$

Denote by $\mathcal{G}_{Z}$ the set of all elements $\sum_{s \in S_{R *} \cap S_{* L}} r_{S} s$ such that $r_{s} \in F \backslash\{0\}$ for all $s \in S_{R *} \cap S_{* L}$. It is easily seen that, if $\left|M_{Z}\right| \geq 1$, then the set $\mathcal{G}_{Z}$ is nonempty and contains only nonzero elements.

Denote by $\mathcal{G}_{L}$ the set of all elements $\sum_{s \in S_{i *} \cap S_{* L}} r_{s} s$ such that $r_{s} \in F \backslash\{0\}$ for all $s \in S_{i *} \cap S_{* L}$, where $i$ runs over all elements of $I \backslash L$ such that $\left|S_{i *} \cap S_{* L}\right|=M_{L}$. If $\left|M_{L}\right| \geq 1$, then the set $\mathcal{G}_{L}$ is nonempty and contains only nonzero elements.

Denote by $\mathcal{G}_{R}$ the set of all elements $\sum_{s \in S_{* \lambda} \cap S_{R_{*}}} r_{s} s$ such that $r_{s} \in F \backslash\{0\}$ for all $s \in S_{* \lambda} \cap S_{R *}$, where $\lambda$ runs over all elements of $\Lambda \backslash R$ such that $\left|S_{* \lambda} \cap S_{R *}\right|=M_{R}$. If $\left|M_{R}\right| \geq 1$, then the set $\mathcal{G}_{R}$ is nonempty and contains only nonzero elements.

Denote by $\mathcal{G}_{G}$ the set of all elements $\sum_{s \in S_{i \lambda}} r_{s} s$ such that $r_{s} \in F \backslash\{0\}$ for all $s \in S_{i \lambda}$, where $i$ runs over all elements of $I \backslash L$ and $\lambda$ runs over all elements of $\Lambda \backslash R$ such that $\left|S_{i \lambda}\right|=M_{G}$. If $\left|M_{G}\right| \geq 1$, then $\mathcal{G}_{G}$ is nonempty and contains only nonzero elements.

Our main theorem completely describes all sets $C\left(g_{1}, \ldots, g_{k}\right)$ in $F_{0}[S]$ with largest weight. Note that the results of [16] did not use max-plus algebras and involved a restriction on the sandwich matrix of the underlying Rees matrix semigroup; examples show that is impossible to drop this restriction. Our new construction involving maxplus algebras is so convenient that the main theorem of this paper completely describes all optimal sets of centroids in the general case of arbitrary Rees matrix semigroups without any restrictions on the sandwich matrices.

THeORem 4.1. Let $C$ be a centroid set $C\left(g_{1}, \ldots, g_{k}\right)$ in $F_{0}[S]$ with largest weight, where $F$ is an idempotent semifield, let $T$ be a Rees matrix semigroup $M^{0}(G ; I, \Lambda ; P)$ over a group $G$ with sandwich matrix $P$, and let $S$ be a finite subsemigroup of $T$. Then the following conditions are satisfied.

(i) $\operatorname{wt}(C)=\max \left\{M_{Z}, M_{L}, M_{R}, M_{G}\right\}$.

(ii) $C$ contains an element of weight wt(C) belonging to $\mathcal{G}_{Z} \cup \mathcal{G}_{L} \cup \mathcal{G}_{R} \cup \mathcal{G}_{G}$.

(iii) $\operatorname{wt}(C(r))=\operatorname{wt}(r)=M_{Z}$ for all $r \in \mathcal{G}_{Z}$.

(iv) $\operatorname{wt}(C(r))=\operatorname{wt}(r)=M_{L}$ for all $r \in \mathcal{G}_{L}$. 
(v) $\operatorname{wt}(C(r))=\operatorname{wt}(r)=M_{R}$ for all $r \in \mathcal{G}_{R}$.

(vi) $\operatorname{wt}(C(r))=\operatorname{wt}(r)=M_{G}$ for all $r \in G_{G}$.

\section{Proofs}

For completeness and the reader's convenience, we begin with a few easy and useful lemmas.

Lemma 5.1. Let $F$ be an idempotent semiring and $x_{1}, \ldots, x_{n} \in F$. Then

$$
x_{1}+\cdots+x_{n}=0 \Longleftrightarrow x_{1}=\cdots=x_{n}=0 .
$$

Proof. Suppose that $x_{1}+\cdots+x_{n}=0$. Then the laws of addition and multiplication in the definition of an idempotent semiring imply that

$$
x_{i}=x_{i}+0=x_{i}+\left(x_{1}+\cdots+x_{n}\right)=x_{1}+\cdots+x_{n}=0
$$

when $i=1, \ldots, n$. The opposite implication is clear.

Every semiring satisfying (5.1) is said to be zero sum free. Thus, Lemma 5.1 tells us that idempotent semirings are zero sum free.

Lemma 5.2. Let $x_{1}, \ldots, x_{n} \in F$ and $s_{1}, \ldots, s_{n} \in S$, where $F$ is an idempotent semiring and $S$ is a semigroup with zero $\theta$. Then

$$
0=\sum_{i=1}^{n} x_{i} s_{i} \in F_{0}[S] \Longleftrightarrow\left(s_{i} \neq \theta \Longrightarrow x_{i}=0\right) .
$$

Proof. We assume that $0=\sum_{i=1}^{n} x_{i} s_{i} \in F_{0}[S]$. Combining like terms, we deduce that $\sum_{i=1}^{n} x_{i} s_{i}=\sum_{s \in S}\left(\sum_{s_{i}=s} x_{i}\right) s$. Hence, fixing any $s \in S \backslash\{\theta\}$, we get $\sum_{s_{i}=s} x_{i}=0$. Lemma 5.1 shows that $x_{i}=0$ when $s_{i}=s$. It follows that $x_{i}=0$ for all $s_{i} \in S \backslash\{\theta\}$, as required. The opposite implication is clear.

When $x=\sum_{s \in S} r_{s} s \in F_{0}[S]$, we write $\operatorname{supp}(x)$ for the set $\left\{s \in S: r_{s} \neq 0\right\}$, which is called the support of $x$. Evidently, $\operatorname{wt}(x)=|\operatorname{supp}(x)|$.

Lemma 5.3. Let $x, y \in F_{0}[S]$, where $F$ is an idempotent semiring and $S$ is a semigroup. Then $\operatorname{supp}(x+y)=\operatorname{supp}(x) \cup \operatorname{supp}(y)$.

Proof. This follows from (2.2) and Lemma 5.1.

Let $S$ be a semigroup with zero $\theta$. The left and right annihilators of $S$ are the sets $\operatorname{Ann}_{\ell}(S)$ and $\operatorname{Ann}_{r}(S)$ given by

$$
\begin{aligned}
& \operatorname{Ann}_{\ell}(S)=\{x \in S: x S=\theta\}, \\
& \operatorname{Ann}_{r}(S)=\{x \in S: S x=\theta\} .
\end{aligned}
$$

Lemma 5.4. Let $T=M^{0}(G ; I, \Lambda ; P)$ be a Rees matrix semigroup over a group $G$ with sandwich matrix $P$ and let $S$ be a subsemigroup of $T$. Then

$$
\begin{aligned}
& \operatorname{Ann}_{r}(S)=S_{R *} \cup\{\theta\}, \\
& \operatorname{Ann}_{\ell}(S)=S_{* L} \cup\{\theta\} .
\end{aligned}
$$


Proof. We prove only the first equality, since the proof of the other is dual. Clearly, $\theta$ belongs to both sides of (5.2). Besides, (2.1) and (4.1) imply that $\operatorname{Ann}_{r}(S) \supseteq S_{R *}$. To prove the opposite inclusion and complete the proof, we argue by contradiction.

If $\operatorname{Ann}_{r}(S) \nsubseteq S_{R^{*}}$, then we can choose $i \in I \backslash R$ and $x=(g ; i, \mu) \in \operatorname{Ann}_{r}(S)$, where $g \in G$ and $\mu \in \Lambda$. Since $x \in S$, we get $S_{i *} \nsubseteq \theta$. Therefore, (4.1) shows that $S_{* \lambda} p_{\lambda i} \nsubseteq \theta$ for some $\lambda \in \Lambda$. Hence, there exist $h \in G$ and $j \in I$ such that $(h ; j, \lambda) x=\left(h p_{\lambda i} g ; j, \mu\right) \neq \theta$. This contradicts the choice of $x$ in $\operatorname{Ann}_{r}(S)$.

For any semiring $F$, the left and right annihilators of $F$ are the sets $\operatorname{Ann}_{\ell}(F)$ and $\operatorname{Ann}_{r}(F)$ given by

$$
\begin{aligned}
& \operatorname{Ann}_{\ell}(F)=\{x \in F: x F=0\}, \\
& \operatorname{Ann}_{r}(F)=\{x \in F: F x=0\} .
\end{aligned}
$$

Lemma 5.5. Let $F$ be an idempotent semifield and $S$ be a semigroup with zero $\theta$. Then

$$
\begin{aligned}
& \operatorname{Ann}_{r}\left(F_{0}[S]\right)=F_{0}\left[\operatorname{Ann}_{r}(S)\right], \\
& \operatorname{Ann}_{\ell}\left(F_{0}[S]\right)=F_{0}\left[\operatorname{Ann}_{\ell}(S)\right] .
\end{aligned}
$$

Proof. Suppose that $x=\sum_{i=1}^{n} x_{i} s_{i}$ and $y=\sum_{j=1}^{m} y_{j} t_{j}$ in $F_{0}[S]$, where $x_{i} \in F \backslash\{0\}$ when $i=1, \ldots, n$ and $y_{j} \in F \backslash\{0\}$ when $j=1, \ldots, m$. The product $x_{i} y_{j}$ is nonzero for all $i$ and $j$, because $F$ is a semifield. Therefore, Lemma 5.2 shows that

$$
x y=0 \Longleftrightarrow s_{i} t_{j}=0 \text { for all } i, j .
$$

Equalities (5.4) and (5.5) follow.

Let $S$ be a subsemigroup of a Rees matrix semigroup $M^{0}(G ; I, \Lambda ; P)$; by definition, $S_{R *}^{0}=S_{R *} \cup\{\theta\}$ and $S_{* L}^{0}=S_{* L} \cup\{\theta\}$. Clearly, $S_{R *}^{0}$ and $S_{* L}^{0}$ are subsemigroups of $S$.

Lemma 5.6. Let $F$ be an idempotent semiring and let $S$ be a subsemigroup of a Rees matrix semigroup $M^{0}(G ; I, \Lambda ; P)$ over a group $G$ with sandwich matrix $P$. Then

$$
\begin{aligned}
& \operatorname{Ann}_{r}\left(F_{0}[S]\right)=F_{0}\left[S_{R *}^{0}\right], \\
& \operatorname{Ann}_{\ell}\left(F_{0}[S]\right)=F_{0}\left[S_{* L}^{0}\right] .
\end{aligned}
$$

Proof. These follow from Lemmas 5.4 and 5.5.

Proof of Theorem 4.1 If $S$ does not contain $\theta$, then we may replace $S$ with $S \cup\{\theta\}$ in the statement of the theorem. This does not change the sets $L$ and $R$, nor the numbers $M_{Z}, M_{L}, M_{R}$, and $M_{G}$. Therefore, we also assume that $S$ contains $\theta$.

First, we prove condition (iii). Take any element $r \in \mathcal{G}_{Z}$. By definition, we may write $r$ as $\sum_{s \in S_{R *} \cap S_{* L}} r_{s} s$, where $r_{s} \in F \backslash\{0\}$ for all $s \in S_{R *} \cap S_{* L}$. Hence, $\operatorname{wt}(r)=M_{Z}$. It follows from the equality (5.6) of Lemma 5.6 that $r \in \operatorname{Ann}_{r}\left(F_{0}[S]\right)$. Equality (5.7) demonstrates that $r \in \operatorname{Ann}_{\ell}\left(F_{0}[S]\right)$. Since $F$ is a semifield, it follows that $C(r)$ coincides with the subsemiring $\{c r: c \in \mathbb{N}\}$ generated by $r$ in $F_{0}[S]$. All elements of this set have the same weight, equal to the weight of $r$. Hence, $\operatorname{wt}(C(r))=\operatorname{wt}(r)$, and condition (iii) holds. 
Next, we prove condition (iv). Choose an arbitrary element $r \in \mathcal{G}_{L}$. Then there exists $i \in I \backslash L$ such that $r=\sum_{s \in S_{i *} \cap S_{* L}} r_{s} s$, where $r_{s} \in F \backslash\{0\}$ for all $s \in S_{i *} \cap S_{* L}$ and $\left|S_{i *} \cap S_{* L}\right|=M_{L}$. Therefore, $\operatorname{wt}(r)=M_{L}$.

To prove that $\operatorname{wt}(C(r))=\mathrm{wt}(r)$, we pick any element $x$ in $C(r)$, and claim that $\operatorname{wt}(x) \geq \operatorname{wt}(r)$.

By (3.1), $x=\sum_{j=1}^{k} a_{j} r b_{j}$ for some $a_{j}, b_{j} \in F_{0}[S]^{1} \cup\{1\}$. Since every nonzero element of $F_{0}[S]$ is the sum of some elements from the set $F S$, equal to

$$
\{f s: f \in F \backslash\{0\}, s \in S \backslash\{\theta\}\},
$$

the distributive law allows us to assume that $a_{j}, b_{j} \in F S \cup\{1\}$. We may assume that all summands $a_{j} r b_{j}$ are nonzero.

Suppose that $b_{j} \neq 1$ for some $j$. Since $\operatorname{supp}(r) \subseteq S_{* L}$, the equality (5.3) in Lemma 5.4 shows that $r b_{j}=0$ and so $a_{j} r b_{j}=0$. Therefore, we may also assume that $b_{j}=1$ when $j=1, \ldots, k$.

In view of Lemma 5.3, it remains to verify that $\operatorname{wt}\left(a_{j} r\right) \geq \operatorname{wt}(r)$ when $j=1, \ldots, k$.

Consider a product $a_{j} r$, where $a_{j} \in F S$, that is, $a_{j}=f s$, where $f \in F$ and $s \in S$. Since $F$ is a semifield, $\operatorname{wt}(f s r)=\operatorname{wt}(s r)$. We can write $s$ as $\left(g ; j^{\prime}, \mu\right)$ for some $j^{\prime} \in I$ and $\mu \in \Lambda$. Since $s r \neq 0$, it follows that $p_{\mu i} \neq \theta$. Hence, it follows from (2.1) that $\operatorname{supp}(s r)=S_{j^{\prime} *} \cap S_{* L}$. Therefore, $|\operatorname{supp}(s r)|=\left|S_{j^{\prime} *} \cap S_{* L}\right|=\left|S_{i *} \cap S_{* L}\right|=\operatorname{supp}(r)$. Thus, $\operatorname{wt}\left(a_{j} r\right) \geq \operatorname{wt}(r)$, and so $\mathrm{wt}(x) \geq \operatorname{wt}(r)$ by Lemma 5.3. It follows immediately that $\operatorname{wt}(C(r))=\operatorname{wt}(r)$, which means that condition (iv) holds.

The proof of condition (v) is dual to that of condition (iv) and we omit it.

We now prove condition (vi). Take any element $r \in \mathcal{G}_{G}$. There exist $i \in I \backslash L$ and $\lambda \in \Lambda \backslash R$ such that $r=\sum_{s \in S_{i \lambda}} r_{s} s$ and $\left|S_{i \lambda}\right|=M_{G}$. Therefore, $\operatorname{supp}(r)=\left|M_{G}\right|$. It remains to prove that $\operatorname{wt}(C(r))=\operatorname{wt}(r)$. To this end, we pick any element $x$ in $C(r)$. By (3.1), we may write $x$ as $\sum_{j=1}^{k} a_{j} r b_{j}$ for some $a_{j}, b_{j} \in F_{0}[S] \cup\{1\}$; the distributive law allows us to assume that $a_{j}, b_{j} \in F S \cup\{1\}$, and we may assume that all summands $a_{j} r b_{j}$ are nonzero.

We claim that $\operatorname{wt}(x) \geq \operatorname{wt}(r)$. Keeping in mind Lemma 5.3, it suffices to verify that $\operatorname{wt}\left(a_{j} r b_{j}\right) \geq \operatorname{wt}(r)$ when $j=1, \ldots, k$. Write $a_{j}=f_{a}\left(g_{a} ; i_{a}, \lambda_{a}\right)$ and $b_{j}=f_{b}\left(g_{b} ; i_{b}, \lambda_{b}\right)$ for some $g_{a}, g_{b} \in G, i_{a}, i_{b} \in I, \lambda_{a}, \lambda_{b} \in \Lambda$, and $f_{a}, f_{b} \in F$. Since $\operatorname{supp}(r) \subseteq S_{i \lambda}$ and $a_{j} r b_{j} \neq 0$, it follows from (2.1) that $p_{\lambda_{a} i}, p_{\lambda i_{b}} \neq \theta$. Now $\left|\operatorname{supp}\left(a_{j} r b_{j}\right)\right|=|\operatorname{supp}(r)|$, again by (2.1), because

$$
\operatorname{supp}\left(a_{j} r b_{j}\right)=\left(g_{a} ; i_{a}, \lambda_{a}\right) \operatorname{supp}(r)\left(g_{b} ; i_{b}, \lambda_{b}\right) .
$$

Thus, $\operatorname{wt}\left(a_{j} r b_{j}\right)=\mathrm{wt}(r)$ in this case.

The cases where $a_{j}=1$ or $b_{j}=1$ are similar and even simpler. In these cases too, $\operatorname{wt}\left(a_{j} r b_{j}\right)=\mathrm{wt}(r)$. Thus, Lemma 5.3 shows that $\mathrm{wt}(C(r))=\mathrm{wt}(r)$, as required. This means that condition (vi) holds.

Now we prove condition (ii). Choose a nonzero element $r$ of minimal weight in $C$ and consider several possible cases.

Case 1: $r \in \operatorname{Ann}_{\ell}\left(F_{0}[S]\right) \cap \operatorname{Ann}_{r}\left(F_{0}[S]\right)$. By Lemma 5.6, $r \in F_{0}\left[\left(S_{R *} \cap S_{* L}\right)^{0}\right]$ and so $\operatorname{supp}(r) \subseteq S_{R *} \cap S_{* L}$. It follows from the maximality of $\operatorname{wt}(C)$ and condition (iii), 
which we have already proved, that $|\operatorname{supp}(r)|=M_{Z}$. Therefore, $\operatorname{supp}(r)=S_{R *} \cap S_{* L}$, whence $r \in \mathcal{G}_{Z}$. Since $\mathrm{wt}(r)=\mathrm{wt}(C)$, condition (ii) holds in this case.

Case 2: $r \in \operatorname{Ann}_{\ell}\left(F_{0}[S]\right) \backslash \operatorname{Ann}_{r}\left(F_{0}[S]\right)$. Equality (5.4) of Lemma 5.5 shows that $\operatorname{supp}(r) \nsubseteq \operatorname{Ann}_{r}(S)$. Hence, there exists $b \in S$ such that $b \operatorname{supp}(r) \neq \theta$. We can write $b$ as $\left(g_{b} ; i_{b}, \lambda_{b}\right)$ for some $g_{b} \in G, i_{b} \in I$, and $\lambda_{b} \in \Lambda$. Here $i_{b} \notin R$, because of the equality (5.2) of Lemma 5.4. It follows from (2.1) that $\operatorname{supp}(b r) \subseteq S_{i_{b} *}$. Since $r \in \operatorname{Ann}_{\ell}\left(F_{0}[S]\right)=S_{* L}$, we deduce that $\operatorname{supp}(b r) \subseteq S_{i_{b} *} \cap S_{* L}$.

Condition (iv) (proved above) shows that $F_{0}[S]$ contains a set $C\left(g_{1}, \ldots, g_{n}\right)$ of weight $M_{L}$. Since $M_{L} \geq\left|S_{i_{b} *} \cap S_{* L}\right|$, the maximality of the weight of $C$ ensures that the weight of every nonzero element in $C$ is at least $M_{L}$. From this, it follows that $\operatorname{wt}(b r)=M_{L}=\left|S_{i_{b} *} \cap S_{* L}\right|$ and $\operatorname{supp}(b r)=S_{i_{b} *} \cap S_{* L}$. This means that $b r \in \mathcal{G}_{L}$.

Now $\operatorname{wt}(b r)=\operatorname{wt}(r)=\operatorname{wt}(C)$, since $b r \in C \backslash\{0\}$ and $r$ has minimal weight in $C$. Thus, condition (ii) holds in this case, too.

Case 3: $r \in \operatorname{Ann}_{r}\left(F_{0}[S]\right) \backslash \operatorname{Ann}_{\ell}\left(F_{0}[S]\right)$. This case is dual to Case 2 and we omit the proof.

Case 4: $r \notin \operatorname{Ann}_{r}\left(F_{0}[S]\right) \cup \operatorname{Ann}_{\ell}\left(F_{0}[S]\right)$. First, $r \notin F_{0}\left[\operatorname{Ann}_{r}(S)\right] \cup F_{0}\left[\operatorname{Ann}_{\ell}(S)\right]$, by Lemma 5.5. Hence, there exist $a, b \in S$ such that $a \operatorname{supp}(r), \operatorname{supp}(r) b \neq \theta$. Then (2.1) shows that $a \operatorname{supp}(r) b \neq \theta$. Since $r$ has minimum weight in $C$ and $\operatorname{arb} \in C \backslash\{0\}$, we deduce that $\operatorname{wt}(a r b)=\operatorname{wt}(r)=\operatorname{wt}(C)$. Consider the representations $a=\left(g_{a} ; i_{a}, \lambda_{a}\right)$ and $b=\left(g_{b} ; i_{b}, \lambda_{b}\right)$, where $g_{a}, g_{b} \in G, i_{a}, i_{b} \in I$, and $\lambda_{a}, \lambda_{b} \in \Lambda$. By (2.1), it follows readily that $\operatorname{supp}(a r b) \subseteq S_{i_{a} \lambda_{b}}$; in view of the maximality of $M_{G}$, we conclude that $\operatorname{supp}(a r b) \leq M_{G}$. Condition (vi) (proved above) tells us that $F_{0}[S]$ contains a set of the form $C\left(g_{1}, \ldots, g_{n}\right)$ with weight $M_{G}$. By the maximality of $\operatorname{wt}(C)$, we deduce that $\operatorname{wt}(a r b) \geq M_{G}$ and hence wt $(a r b)=M_{G}$. Therefore, $a r b \in \mathcal{G}_{G}$, and condition (ii) holds in this case, too.

Finally, we prove condition (i). Clearly, condition (ii) implies that

$$
\operatorname{wt}(C) \leq \max \left\{M_{Z}, M_{L}, M_{R}, M_{G}\right\} .
$$

On the other hand, the maximality of $\operatorname{wt}(C)$ and conditions (iii) to (vi) show that $\operatorname{wt}(C) \geq \max \left\{M_{Z}, M_{L}, M_{R}, M_{G}\right\}$. Therefore, condition (i) is satisfied.

\section{Acknowledgement}

The authors are grateful to the referee for helpful corrections.

\section{References}

[1] F. Baccelli, G. Cohen, G. J. Olsder and J.-E. Quadrat, Synchronization and Linearity: An Algebra for Discrete Event Systems (Wiley Interscience, New York, 1992).

[2] A. M. Bagirov and J. L. Yearwood, 'A new nonsmooth optimization algorithm for minimum sumof-squares clustering problems', European J. Oper. Res. 170 (2006), 578-596.

[3] D. M. Clark and B. A. Davey, Natural Dualities for the Working Algebraist (Cambridge University Press, Cambridge, 1998). 
[4] B. A. Davey, M. Jackson, M. Maróti and R. N. McKenzie, 'Principal and syntactic congruences in congruence-distributive and congruence-permutable varieties', J. Aust. Math. Soc. 85 (2008), $59-74$.

[5] R. Dazeley, J. L. Yearwood, B. H. Kang and A. V. Kelarev, 'Consensus clustering and supervised classification for profiling phishing emails in internet commerce security', Lecture Notes in Comput. Sci. 6232 (2010), 235-246.

[6] D. Easdown, J. East and D. G. FitzGerald, 'A presentation of the dual symmetric inverse monoid', Internat. J. Algebra Comput. 18 (2008), 357-374.

[7] D. Easdown and W. D. Munn, 'Trace functions on inverse semigroup algebras', Bull. Aust. Math. Soc. 52 (1995), 359-372.

[8] J. S. Golan, Semirings and Their Applications (Kluwer Academic, Dordrecht, 1999).

[9] J. M. Howie, Fundamentals of Semigroup Theory (Clarendon Press, Oxford, 1995).

[10] M. Jackson, 'The embeddability of ring and semigroup amalgams is undecidable', J. Aust. Math. Soc. 69 (2000), 272-286.

[11] M. Jackson, 'On the finite basis problem for finite Rees quotients of free monoids', Acta Sci. Math. (Szeged) 67 (2001), 121-159.

[12] M. Jackson and M. Volkov, 'Undecidable problems for completely 0-simple semigroups', J. Pure Appl. Algebra 213(10) (2009), 1961-1978.

[13] A. V. Kelarev, Ring Constructions and Applications (World Scientific, River Edge, NJ, 2002).

[14] A. V. Kelarev, Graph Algebras and Automata (Marcel Dekker, New York, 2003).

[15] A. V. Kelarev, R. Göbel, K. M. Rangaswamy, P. Schultz and C. Vinsonhaler, Abelian Groups, Rings and Modules, Contemporary Mathematics 273 (American Mathematical Society, New York, 2001).

[16] A. V. Kelarev, P. W. Watters and J. L. Yearwood, 'Rees matrix constructions for clustering of data', J. Aust. Math. Soc. 87 (2009), 377-393.

[17] A. V. Kelarev, J. L. Yearwood and M. A. Mammadov, 'A formula for multiple classifiers in data mining based on Brandt semigroups', Semigroup Forum 78(2) (2009), 293-309.

[18] A. V. Kelarev, J. L. Yearwood and P. W. Vamplew, 'A polynomial ring construction for classification of data', Bull. Aust. Math. Soc. 79 (2009), 213-225.

[19] A. V. Kelarev, J. L. Yearwood, P. Watters, X. W. Wu, J. H. Abawajy and L. Pan, 'Internet security applications of the Munn rings', Semigroup Forum 81(1) (2010), 162-171.

[20] I. H. Witten and E. Frank, Data Mining: Practical Machine Learning Tools and Techniques (Elsevier/Morgan Kaufman, Amsterdam, 2005).

[21] J. Yearwood, D. Webb, L. Ma, P. Vamplew, B. Ofoghi and A. Kelarev, 'Applying clustering and ensemble clustering approaches to phishing profiling', Proc. 8th Australasian Data Mining Conf., Data Mining and Analytics 2009 (ausDM 2009), Melbourne, Australia, 1-4 December 2009, CRPIT, Vol. 101, pp. 25-34.

\section{A. V. KELAREV, School of Science, Information Technology and Engineering, University of Ballarat, PO Box 663, Ballarat, Victoria 3353, Australia e-mail: a.kelarev@ballarat.edu.au}

\section{J. L. YEARWOOD, School of Science, Information Technology and Engineering, University of Ballarat, PO Box 663, Ballarat, Victoria 3353, Australia e-mail: j.yearwood@ballarat.edu.au}

\section{LIFANG ZI, School of Science, Information Technology and Engineering, University of Ballarat, PO Box 663, Ballarat, Victoria 3353, Australia e-mail: 1.zi@ballarat.edu.au}

